\title{
Deligne Products of Line Bundles over Moduli Spaces of Curves
}

\author{
L. Weng ${ }^{1, \star}$, D. Zagier $^{2,3}$ \\ 1 Graduate School of Mathematics, Kyushu University, Fukuoka, Japan. \\ E-mail: weng@math.kyushu-u.ac.jp \\ 2 Max-Planck-Institut für Mathematik, Bonn, Germany \\ 3 Collège de France, Paris, France
}

Received: 22 June 2007 / Accepted: 9 October 2007

Published online: 20 May 2008 - (C) The Author(s) 2008

Abstract: We study Deligne products for forgetful maps between moduli spaces of marked curves by offering a closed formula for tautological line bundles associated to marked points. In particular, we show that the Deligne products for line bundles on the total spaces corresponding to "forgotten" marked points are positive integral multiples of the Weil-Petersson bundles on the base moduli spaces.

\section{Introduction}

Let $\mathcal{M}_{g, N}$ denote the moduli space of curves $C$ of genus $g$ with $N$ ordered marked points $P_{1}, \ldots, P_{N}$, and $\pi=\pi_{N}: \mathcal{C}_{g, N} \rightarrow \mathcal{M}_{g, N}$ the universal curve over $\mathcal{M}_{g, N}$. (We are using the language of stacks here [3].) The marked points give sections $\mathbf{P}_{i}: \mathcal{M}_{g, N} \rightarrow$ $\mathcal{C}_{g, N}, i=1, \ldots, N$ of $\pi$.

The Picard group of $\mathcal{M}_{g, N}$ is known to be free of rank $N+1$ [4] and has a $\mathbb{Z}$-basis given by the Mumford class $\lambda$ (the line bundle whose fiber at $C$ is $\operatorname{det} H^{0}\left(C, K_{C}\right) \otimes$ $\left.\operatorname{det} H^{1}\left(C, K_{C}\right)^{-1}\right)$ and the "tautological line bundles" $\ell_{i}:=\mathbf{P}_{i}^{*}\left(K_{N}\right)$, where $K_{N}$ is the relative canonical line bundle (relative dualizing sheaf) of $\pi[1,5]$. The $\ell_{i}$ carry metrics in such a way that their first Chern forms give the Kähler metrics on $\mathcal{M}_{g, N}$ defined by Takhtajan-Zograf $[9,10]$ in terms of Eisenstein series associated to punctured Riemann surfaces $[11,12]$. There is a further interesting element $\Delta \in \operatorname{Pic}\left(\mathcal{M}_{g, N}\right)$, whose associated first Chern form (for a certain natural metric) gives the Kähler form for the Weil-Petersson metric on $\mathcal{M}_{g, N}[11,12]$; it is given in terms of $\lambda$ and the $\ell_{i}$ by the Riemann-Roch formula $\Delta=12 \lambda+\sum_{i=1}^{N} \ell_{i}$. We can also define $\Delta$ by the formula $\Delta:=\left\langle K_{N}\left(\mathbf{P}_{1}+\cdots+\mathbf{P}_{N}\right), K_{N}\left(\mathbf{P}_{1}+\cdots+\mathbf{P}_{N}\right)\right\rangle_{\pi}$, where $\langle\cdot, \cdot\rangle_{\pi}: \operatorname{Pic}\left(\mathcal{C}_{g, N}\right)^{2} \rightarrow$ $\operatorname{Pic}\left(\mathcal{M}_{g, N}\right)$ denotes the Deligne pairing. We recall that the Deligne pairing is a bilinear $\operatorname{map}\langle\cdot, \cdot\rangle_{p}: \operatorname{Pic}(Y)^{2} \rightarrow \operatorname{Pic}(X)$ which is defined for any flat morphism $p: Y \rightarrow X$

\footnotetext{
^ Partially supported by the Japan Society for the Promotion of Science.
} 
of relative dimension 1 and that it can be generalized to a multilinear map $\langle\cdot, \ldots, \cdot\rangle_{p}$ : $\operatorname{Pic}(Y)^{n+1} \rightarrow \operatorname{Pic}(X)$, the Deligne product, defined for any flat morphism $p: Y \rightarrow X$ of relative dimension $n$. (The precise definitions will be given in §3.)

We are interested in computing the Deligne product explicitly for the forgetful map

$$
\pi_{N, m}: \mathcal{M}_{g, N+m} \rightarrow \mathcal{M}_{g, N}, \quad\left(C ; P_{1}, \ldots, P_{N+m}\right) \mapsto\left(C ; P_{1}, \ldots, P_{N}\right) .
$$

In other words, if we use $\tilde{\lambda}, \tilde{\Delta}, \tilde{\ell}_{j}$ to denote the Mumford, Weil-Petersson and tautological line bundles on $\mathcal{M}_{g, N+m}$, respectively, then we would like to compute $\left\langle L_{1}, \ldots, L_{m+1}\right\rangle_{\pi_{N, m}}$ as a linear combination of $\ell_{1}, \ldots, \ell_{N}$ and $\lambda$ (or $\Delta$ ) on $\mathcal{M}_{g, N}$, where each $L_{v}$ is one of $\tilde{\ell}_{1}, \ldots, \tilde{\ell}_{N+m}$ and $\tilde{\lambda}$ (or $\tilde{\Delta}$ ). We have not solved this problem in general (though it is interesting and perhaps not intractable), but only in the case where each $L_{v}$ is one of the $\tilde{\ell}_{i}$, i.e., where $\tilde{\lambda}$ (or $\tilde{\Delta}$ ) does not appear. The formula we find expresses $\left\langle L_{1}, \ldots, L_{m+1}\right\rangle_{\pi_{N, m}}$ in this case as a positive linear combination of $\Delta$ and those $\ell_{i}(i=1, \ldots, N)$ for which $\tilde{\ell}_{i}$ appear among the $L_{v}$. In particular, if each of $L_{1}, \ldots, L_{m+1}$ is one of the last $m$ line bundles $\tilde{\ell}_{N+1}, \ldots, \tilde{\ell}_{N+m}$, then $\left\langle L_{1}, \ldots, L_{m+1}\right\rangle_{\pi_{N, m}}$ is simply a positive integer multiple of the Weil-Petersson class $\Delta$, giving an interesting relation between the Weil-Petersson and the tautological line bundles.

\section{Statement of the Theorem}

As just explained, we want to compute the Deligne product $\left\langle L_{1}, \ldots, L_{m+1}\right\rangle_{\pi_{N, m}}$, where each $L_{v}$ belongs to the set $\left\{\tilde{\ell}_{1}, \ldots, \tilde{\ell}_{N+m}\right\}$. It turns out to be more convenient to use the multiplicities where the $\tilde{\ell}_{i}$ occur in $\left\{L_{1}, \ldots, L_{m+1}\right\}$ as coordinates. We therefore introduce the notation

$$
\begin{aligned}
T_{N, m}\left(a_{1}, \ldots, a_{N+m}\right):= & \langle\underbrace{\tilde{\ell}_{1}, \ldots, \tilde{\ell}_{1}}_{a_{1}}, \ldots, \underbrace{\tilde{\ell}_{N+m}, \ldots, \tilde{\ell}_{N+m}}_{a_{N+m}}\rangle_{\pi_{N, m}} \\
& \in \operatorname{Pic}\left(\mathcal{M}_{g, N}\right),
\end{aligned}
$$

where $a_{1}, \ldots, a_{N+m} \in \mathbb{Z}_{\geq 0}$ with $a_{1}+\cdots+a_{N+m}=m+1$. We will sometimes denote this element by $T_{N, m}\left(a_{1}, \ldots, a_{N} ; a_{N+1}, \ldots, a_{N+m}\right)$ or even, setting $a_{N+i}=: d_{i}$, by $T_{N, m}\left(a_{1}, \ldots, a_{N} ; d_{1}, \ldots, d_{m}\right)$, to emphasize the different roles played by the indices corresponding to the points which are also marked in $\mathcal{M}_{g, N}$ and to those which are "forgotten" by the projection map $\pi_{N, m}$. Our main result is then:

Theorem. Let $a_{1}, \ldots, a_{N}, d_{1}, \ldots, d_{m} \geq 0$ be non-negative integers with sum $m+1$. Then the line bundle $T_{N, m}\left(a_{1}, \ldots, a_{N} ; \bar{d}_{1}, \ldots, d_{m}\right)$ defined in (1) is given in terms of the elements $\ell_{i}, \Delta \in \operatorname{Pic}\left(\mathcal{M}_{g, N}\right)$ by the formula

$$
\begin{aligned}
& \left(\prod_{i=1}^{N} a_{i} !\right)\left(\prod_{j=1}^{m} d_{j} !\right) T_{N, m}\left(a_{1}, \ldots, a_{N} ; d_{1}, \ldots, d_{m}\right) \\
& =C_{1}(\mathbf{d}) \sum_{i=1}^{N} a_{i}\left(\ell_{i}-\frac{\Delta}{\widetilde{N}}\right)+C_{2}(\mathbf{d}) \Delta,
\end{aligned}
$$


where $\widetilde{N}=N+2 g-3$ and the coefficients $C_{v}(\mathbf{d})=C_{v}\left(\widetilde{N}, d_{1}, \ldots, d_{m}\right)(v=1,2)$ depend only on the $d_{i}$ and on $\widetilde{N}$ and are given explicitly by

$$
\begin{aligned}
C_{1}(\mathbf{d}) & =\sum_{n=0}^{m} \frac{(m-n) !(\tilde{N}+n-1) !}{(\widetilde{N}-1) !} \sigma_{n}, \\
C_{2}(\mathbf{d}) & =\sum_{n=0}^{m} \frac{(m-n+1) !(\widetilde{N}+n-2) !}{\widetilde{N}(\widetilde{N}-2) !} \sigma_{n}
\end{aligned}
$$

with $\sigma_{n}=\sigma_{n}\left(d_{1}, \ldots, d_{m}\right)$ the $n^{\text {th }}$ elementary symmetric polynomial in the $d_{i}$.

Remark. 1. If $\widetilde{N}=0$ or 1 , then the factors $1 /(\widetilde{N}-1)$ ! and $1 / \widetilde{N}(\widetilde{N}-2)$ ! occurring in the formulas for $C_{1}(\mathbf{d})$ and $C_{2}(\mathbf{d})$ are to be interpreted as $\widetilde{N} / \widetilde{N}$ ! and $(\widetilde{N}-1) / \widetilde{N}$ !, respectively.

2. The proof (or rather, the recursive description of the $T_{N, m}$ on which it is based) will show that in the formula for $T_{N, m}\left(a_{1}, \ldots, a_{N} ; d_{1}, \ldots, d_{m}\right)$ in terms of $\ell_{i}$ and $\Delta$, all the coefficients are non-negative and integral (even though $\ell_{1}, \ldots, \ell_{N}, \Delta$ is not a $\mathbb{Z}$-basis of $\left.\operatorname{Pic}\left(\mathcal{M}_{g, N}\right)\right)$. Neither property is obvious from the formulas (2) and (3), though one can see easily that both $C_{1}(\mathbf{d})$ and $C_{2}(\mathbf{d})-\left(m+1-\sigma_{1}\right) C_{1}(\mathbf{d}) / \widetilde{N}$, the coefficient of $\Delta$ on the right-hand side of (2), are polynomials in $\widetilde{N}$.

3. In Sect. 6 we will give an alternative explicit formula for the coefficients $C_{1}(\mathbf{d})$ and $\mathrm{C}_{2}(\mathbf{d})$.

4. Notice that, as already mentioned in the Introduction, formula (2) in the special case when all the $a_{i}$ are 0 says that $T_{N, m}\left(0, \ldots, 0 ; d_{1}, \ldots, d_{m}\right)$ is a multiple of $\Delta$ alone. In other words, all Deligne products of line bundles corresponding to points which are "forgotten" by $\pi_{N, m}$ are positive integral multiples of the Weil-Petersson bundle $\Delta$.

\section{The Deligne product}

We start with some basic facts about Deligne products [2]. Let $\pi: X \rightarrow S$ be a flat family of algebraic varieties of relative dimension $n$. Then for any $n+1$ invertible sheaves $L_{0}, \ldots, L_{n}$ over $X$, following Deligne [2], we may introduce the Deligne prod$u c t$, denoted by $\left\langle L_{0}, \ldots, L_{n}\right\rangle(X / S)$ or $\left\langle L_{0}, \ldots, L_{n}\right\rangle_{\pi}$ or simply $\left\langle L_{0}, \ldots, L_{n}\right\rangle$, defined uniquely by the following axioms:

(DP1) $\left\langle L_{0}, \ldots, L_{n}\right\rangle(X / S)$ is an invertible sheaf on $S$, and is symmetric and multi-linear in the $L_{i}$ 's.

(DP2) $\left\langle L_{0}, \ldots, L_{n}\right\rangle(X / S)$ is locally generated by the symbols $\left\langle t_{0}, \ldots, t_{n}\right\rangle$, where the $t_{i}$ are sections of $L_{i}$ whose divisors have no common intersection, and these symbols satisfy the following property: if one multiplies one of the sections $t_{i}$ by a rational function $f$ on $X$, where $\bigcap_{j \neq i} \operatorname{div}\left(t_{j}\right)=\sum n_{k} Y_{k}$ is finite over $S$ and $\operatorname{div}(f)$ has no intersection with any $Y_{k}$, then

$$
\left\langle t_{0}, \ldots, f t_{i}, \ldots, t_{n}\right\rangle=\left(\prod_{k} \operatorname{Norm}_{Y_{k} / S}(f)^{n_{k}}\right) \cdot\left\langle t_{0}, \ldots, t_{n}\right\rangle .
$$

(Here $\operatorname{Norm}_{Y_{k} / S}(f)$ is defined as follows: Since $Y_{k}$ is finite over $S$, the function field of $Y_{k}$ is a finite extension of that of $S$, and hence can be viewed as a finite dimensional vector space. Since $f$ is in the function field of $X$, via restriction we may view $f$ as an 
element of the function field of $Y_{k}$. Then multiplication by $f$ defines a linear map of the vector space of the function field of $Y_{k}$ over the function field of $S$. By definition, the determinant of this linear map is called the norm of $f$ with respect to $Y_{k} / S$.)

(DP3) If $t_{n}$ is a section of $L_{n}$ such that all components $D_{\alpha}$ of the $\operatorname{divisor} \operatorname{div}\left(t_{n}\right)=$ $\sum_{\alpha} n_{\alpha} D_{\alpha}$ are flat (of relative dimension $n-1$ ) over $S$, then we have a canonical isomorphism

$$
\left\langle L_{0}, \ldots, L_{n}\right\rangle(X / S) \simeq\left\langle L_{0}, \ldots, L_{n-1}\right\rangle\left(\operatorname{div}\left(t_{n}\right) / S\right):=\otimes_{\alpha}\left\langle L_{0}, \ldots, L_{n-1}\right\rangle\left(D_{\alpha} / S\right)^{\otimes n_{\alpha}}
$$

Roughly speaking, the Deligne product may be built up as follows using the above axioms: one first uses (DP3) to make an induction on the relative dimension so as to reduce to special cases, say, $n=1$, by using a certain choice of sections, and then shows with the help of axiom (DP2) that this construction does not depend on the choice of sections of line bundles and is symmetric by virtue of the Weil reciprocity law.

As a consequence of these axioms and the uniqueness, we know that the Deligne product formalism is compatible with any base change, and that the products satisfy the following compatibility relations with respect to compositions of flat morphisms:

Proposition 1. ([2]). Let $f: X \rightarrow Y$ and $g: Y \rightarrow Z$ be flat morphisms of relative dimension $n$ and $m$, respectively. Then:

(a) For invertible sheaves $L_{0}, \ldots, L_{n}$ on $X$ and $H_{1}, \ldots, H_{m}$ on $Y$, we have

$$
\left\langle\left\langle L_{0}, \ldots, L_{n}\right\rangle_{f}, H_{1}, \ldots, H_{m}\right\rangle_{g} \simeq\left\langle L_{0}, \ldots, L_{n}, f^{*} H_{1}, \ldots, f^{*} H_{m}\right\rangle_{g \circ f} .
$$

(b) For invertible sheaves $L_{1}, \ldots, L_{n}$ on $X$ and $H_{0}, \ldots, H_{m}$ on $Y$, we have

$$
\left\langle\left\langle f^{*} H_{0}, L_{1} \ldots, L_{n}\right\rangle_{f}, H_{1}, \ldots, H_{m}\right\rangle_{g} \simeq\left\langle H_{0}, H_{1}, \ldots, H_{m}\right\rangle_{g}^{f_{*}\left(c_{1}\left(L_{1}\right) \cdots c_{1}\left(L_{n}\right)\right)} .
$$

A special case of Proposition 1 which will be needed later is the formula

$$
\left\langle L, f^{*} H_{0}, \ldots, f^{*} H_{m}\right\rangle_{g \circ f} \simeq \operatorname{deg}_{f}(L)\left\langle H_{0}, \ldots, H_{m}\right\rangle_{g}
$$

for $f, g$ as in the proposition with $n=1$ and for any bundles $L$ on $X$ and $H_{0}, \ldots, H_{m}$ on $Y$. To get this, we use part (a) of the proposition to write the left-hand side as $\left\langle\left\langle L, f^{*} H_{0}\right\rangle_{f}, H_{1}, \ldots, H_{m}\right\rangle_{g}$ and then part (b) to write $\left\langle L, f^{*} H_{0}\right\rangle_{f}$ as $\operatorname{deg}_{f}(L) H_{0}$.

Remark. Recall that there is a map $c_{1}$ from $\operatorname{Pic}(X)$ to the codimension 1 part $\mathrm{CH}^{1}(X)$ of the Chow group of $X$. If $f: X \rightarrow Y$ is flat of relative dimension $n$ and $L_{0}, \ldots, L_{n}$ belong to $\operatorname{Pic}(X)$, then the image of $\left\langle L_{0}, \ldots, L_{n}\right\rangle$ under $c_{1}$ is equal to the image of the product $c_{1}\left(L_{0}\right) \cdots c_{1}\left(L_{n}\right)$ under the push-forward map $f_{*}: \mathrm{CH}^{n+1}(X) \rightarrow \mathrm{CH}^{1}(Y)$. At this level, formulas (4a), (4b) and (5) are just specializations of the general projection formula $g_{*}\left(f_{*}(A) \cdot B\right)=(g f)_{*}\left(A \cdot f^{*}(B)\right)$, valid for any flat morphisms $f: X \rightarrow Y$ and $g: Y \rightarrow Z$ and elements $A \in \mathrm{CH}(X), B \in \mathrm{CH}(Y)$. 


\section{Geometric Preparations}

We now apply the Deligne product to universal curves over moduli spaces. As in $\S 1$, we denote by $K_{N}$ the relative canonical line bundle of $\pi=\pi_{N}: \mathcal{C}_{g, N} \rightarrow \mathcal{M}_{g, N}$, by $\mathbf{P}_{i}$ $(1 \leq i \leq N)$ the $N$ sections of $\pi$ and by $\ell_{i}=\mathbf{P}_{i}^{*}\left(K_{N}\right)$ the $i^{\text {th }}$ tautological line bundle on $\mathcal{M}_{g, N}$. We write $L_{i}(1 \leq i \leq N+1)$ for the line bundles on $\mathcal{C}_{g, N}$ defined in the same way, where $\mathcal{C}_{g, N}$ is identified with $\mathcal{M}_{g, N+1}$. Also for convenience, we denote the bundle $\mathcal{O}_{\mathcal{C}_{g, N}}\left(\mathbf{P}_{i}\right)(1 \leq i \leq N)$ simply by $\mathbf{P}_{i}$. The following properties can be found in [6,7]. (Deligne products are not used in Knudsen's original papers, but the verbatim change is rather trivial. See e.g., [11,12].)

Proposition 2. ([6]) With the above notations, we have

(a) $\left\langle\mathbf{P}_{i}, \mathbf{P}_{j}\right\rangle_{\pi} \simeq \mathcal{O} \quad(i, j=1, \ldots, N, i \neq j)$;

(b) $\left\langle K_{N}\left(\mathbf{P}_{i}\right), \mathbf{P}_{i}\right\rangle_{\pi} \simeq \mathcal{O} \quad(i=1, \ldots, N)$;

(c) $L_{i} \simeq \pi^{*} \ell_{i}+\mathbf{P}_{i} \quad(i=1, \ldots, N)$;

(d) $L_{N+1} \simeq K_{N}\left(\mathbf{P}_{1}+\cdots+\mathbf{P}_{N}\right)$.

The next proposition, which is a slight extension of Proposition 2, contains all the geometric information which we will need to compute the Deligne products in (1). We use the same notations as above, but also denote by $\pi^{\prime}=\pi_{N-m, m}$ the forgetful map from $\mathcal{M}_{g, N}$ to $\mathcal{M}_{g, N-m}$ for some $m \geq 0$ and use $\xi_{1}, \ldots, \xi_{m}$ to denote $m$ general elements of $\operatorname{Pic}\left(\mathcal{C}_{g, N}\right)$.

Proposition 3. With the above notations, we have

(a) $\left\langle\mathbf{P}_{i}, \mathbf{P}_{j}, \xi_{1}, \ldots, \xi_{m}\right\rangle_{\pi^{\prime} \circ \pi} \simeq \mathcal{O} \quad(i, j=1, \ldots, N, \quad i \neq j)$;

(b) $\left\langle\mathbf{P}_{i}, L_{i}, \xi_{1}, \ldots, \xi_{m}\right\rangle_{\pi^{\prime} \circ \pi} \simeq \mathcal{O} \quad(i=1, \ldots, N)$;

(c) $\left\langle\mathbf{P}_{i}, L_{N+1}\right\rangle_{\pi} \simeq \mathcal{O} \quad(i=1, \ldots, N)$;

(d) $\operatorname{deg}_{\pi}\left(L_{N+1}\right)=2 g-2+N$.

Proof. Since the sections $\mathbf{P}_{i}$ and $\mathbf{P}_{j}$ are disjoint for $i \neq j$, the pull-back of $\mathcal{O}\left(\mathbf{P}_{i}\right)$ to $\mathbf{P}_{j}$ is trivial (Prop. 2(a)). Therefore axiom (DP3) from $\S 3$ implies (a). Next, we use Prop. 2(c) to write

$$
\left\langle L_{i}, \mathbf{P}_{i}, \xi_{1}, \ldots, \xi_{m}\right\rangle_{\pi^{\prime} \circ \pi} \simeq\left\langle\pi^{*} \ell_{i}, \mathbf{P}_{i}, \xi_{1}, \ldots, \xi_{m}\right\rangle_{\pi^{\prime} \circ \pi}+\left\langle\mathbf{P}_{i}, \mathbf{P}_{i}, \xi_{1}, \ldots, \xi_{m}\right\rangle_{\pi^{\prime} \circ \pi} .
$$

By (DP3), the first term equals $\left\langle\ell_{i},\left.\xi_{1}\right|_{\mathbf{P}_{i}}, \ldots,\left.\left.\xi_{m}\right|_{\mathbf{P}_{i}}\right|_{\pi^{\prime}}\right.$ (actually multiplied by $\operatorname{deg}_{\pi}\left(\mathbf{P}_{i}\right)$, but the relative degree of a section is 1$)$, while the second term is $-\left\langle\ell_{i},\left.\xi_{1}\right|_{\mathbf{P}_{i}}, \ldots,\left.\xi_{m}\right|_{\mathbf{P}_{i}}\right\rangle_{\pi^{\prime}}$ by Prop. 2(b) (adjunction formula). This proves (b). Part (c) follows from Prop. 2(d), since $\left\langle\mathbf{P}_{i}, K_{N}\left(\mathbf{P}_{i}\right)\right\rangle_{\pi}$ vanishes by the adjunction formula and all $\left\langle\mathbf{P}_{i}, \mathbf{P}_{j}\right\rangle_{\pi}$ with $j \neq i$ vanish by (a). Part (d) also follows from Prop. 2(d) by taking the relative degree of both sides. We also mention the stronger statement that $\left\langle L_{N+1}, L_{i}\right\rangle_{\pi} \simeq(2 g-2+N) \ell_{i}$ for $i=1, \ldots, N$. The proof of this is similar to the other parts of the proposition, but we omit it since this result will not be used in the sequel.

\section{The Recursion Formula for $T_{N, m}$}

In this section, we use the results of $\S 4$ to give a recursion formula and initial data for the line bundles (1) which determine them completely in $\operatorname{Pic}\left(\mathcal{M}_{g, N}\right)$. These recursions will be solved in $\S 6$.

The recursion formula which we will prove for the $T_{N, m}$ is as follows. 
Proposition 4. (String Equation) For $m \geq 0$ and any integers $a_{1}, \ldots, a_{N+m} \geq 0$, we have

$$
T_{N, m+1}\left(a_{1}, \ldots, a_{N+m}, 0\right)=\sum_{i=1}^{N+m} T_{N, m}\left(a_{1}, \ldots, a_{i}-1, \ldots, a_{N+m}\right),
$$

with the convention that $T_{N, m+1}\left(a_{1}, \ldots, a_{N+m}\right)=0$ if any $a_{i}<0$.

Recall that the indices $a_{i}$ with $i>N$ in $T_{N, m}\left(a_{1}, \ldots, a_{N+m}\right)$ play a different role than the $a_{i}$ with $i \leq N$ and that we also use the notations $d_{j}$ for $a_{N+j}(1 \leq j \leq m)$ and $T_{N, m}\left(a_{1}, \ldots, a_{N} ; d_{1}, \ldots, d_{m}\right)$ for $T_{N, m}\left(a_{1}, \ldots, a_{N+m}\right)$. Proposition 4 lets us reduce the calculation of these bundles by induction to the case when every $d_{i}$ is strictly positive. (If any $d_{j}$ is zero, we can put it in the last position, because $T_{N, m}$ is symmetric in the $d$ 's.) But since $\sum_{i=1}^{N} a_{i}+\sum_{j=1}^{m} d_{j}=m+1$, this can only happen if $\left(d_{1}, \ldots, d_{m}\right)=(1, \ldots, 1)$ or $(2,1, \ldots, 1)$. There are therefore only two initial cases which have to be considered. The values of $T_{N, m}$ in these two cases are given by the following:

Proposition 5. The line bundles $T_{N, m}\left(a_{1}, \ldots, a_{N} ; d_{1}, \ldots, d_{m}\right)$ in the two cases when all the $d_{i}$ are strictly positive are given by the formulas

$$
T_{N, m}(1, \underbrace{0, \ldots, 0}_{N-1} ; \underbrace{1, \ldots, 1}_{m})=\frac{(\tilde{N}+m) !}{\widetilde{N} !} \ell_{1} \quad(m \geq 0)
$$

and

$$
T_{N, m}(\underbrace{0, \ldots, 0}_{N} ; 2, \underbrace{1, \ldots, 1}_{m-1})=\frac{(\tilde{N}+m) !}{(\tilde{N}+1) !} \Delta \quad(m \geq 1),
$$

where $\widetilde{N}=N+2 g-3$.

Proposition 5 in turn can be deduced by induction over $m$ from the special cases

$$
T_{N, 0}(1, \underbrace{0, \ldots, 0}_{N-1} ;)=\ell_{1}, \quad T_{N, 1}(\underbrace{0, \ldots, 0}_{N} ; 2)=\Delta
$$

(the first of which is trivial because the Deligne product is simply the identity map, and the second by the very definition of $\Delta$ ) and from the following companion result to Proposition 4.

Proposition 6. (Dilaton Equation) For $m \geq 0$ and any integers $a_{1}, \ldots, a_{N+m} \geq 0$, we have

$$
T_{N, m+1}\left(a_{1}, \ldots, a_{N+m}, 1\right)=(N+m+2 g-2) T_{N, m}\left(a_{1}, \ldots, a_{N+m}\right) .
$$

The proofs of Propositions 4 and 6 are similar to one another and will be given together. For convenience, we use the abbreviated notation $\left\langle S_{1}^{\circ k_{1}}, S_{2}^{\circ k_{2}}, \ldots, S_{n}^{\circ k_{n}}\right\rangle_{f}$ to denote the Deligne product

$$
\langle\underbrace{S_{1}, \ldots, S_{1}}_{k_{1}}, \underbrace{S_{2}, \ldots, S_{2}}_{k_{2}}, \ldots, \underbrace{S_{n}, \ldots, S_{n}}_{k_{n}}\rangle_{f}
$$


for any line bundles $S_{i}$ and any integers $k_{i} \geq 0$. We also replace the " $N$ " of $\S 4$ by " $N+m$ " and use the same conventions as there, i.e., $\ell_{i}(1 \leq i \leq N+m)$ denotes the $i^{\text {th }}$ tautological line bundle on $\mathcal{M}_{g, N+m}$ and $L_{i}(1 \leq i \leq N+m+1)$ the $i^{\text {th }}$ tautological line bundle on $\mathcal{M}_{g, N+m+1}$, while $\pi$ and $\pi^{\prime}$ denote the projections from $\mathcal{M}_{g, N+m+1}$ to $\mathcal{M}_{g, N+m}$ and from $\mathcal{M}_{g, N+m}$ to $\mathcal{M}_{g, N}$, respectively. Finally, we set $M=N+m$. With these notations, the two formulas to be proved become

$$
\left\langle L_{1}^{\circ a_{1}}, \ldots, L_{M}^{\circ a_{M}}\right\rangle_{\pi^{\prime} \circ \pi}=\sum_{i=1}^{M}\left\langle\ell_{1}^{\circ a_{1}}, \ldots, \ell_{i}^{\circ\left(a_{i}-1\right)}, \ldots, \ell_{M}^{\circ a_{M}}\right\rangle_{\pi^{\prime}}
$$

(with the usual convention that $\left\langle\cdots, \ell_{i}^{\circ\left(a_{i}-1\right)}, \cdots\right\rangle=0$ if $a_{i}=0$ ) and

$$
\left\langle L_{1}^{\circ a_{1}}, \ldots, L_{M}^{\circ a_{M}}, L_{M+1}\right\rangle_{\pi^{\prime} \circ \pi}=(2 g-2+M)\left\langle\ell_{1}^{\circ a_{1}}, \ldots, \ell_{M}^{\circ a_{M}}\right\rangle_{\pi^{\prime}} .
$$

To prove these equations, we proceed as follows. Using Prop. 2(c), Prop. 3(b) and then Prop. 2(c) again (all of them with $N$ replaced by $M$ ), we obtain

$$
\begin{aligned}
\left\langle L_{1}^{\circ a_{1}}, \xi_{1}, \ldots, \xi_{r}\right\rangle_{\pi^{\prime} \circ \pi}= & \left\langle L_{1},\left(\pi^{*} \ell_{1}+\mathbf{P}_{1}\right)^{\circ\left(a_{1}-1\right)}, \xi_{1}, \ldots, \xi_{r}\right\rangle_{\pi^{\prime} \circ \pi} \\
= & \left\langle L_{1},\left(\pi^{*} \ell_{1}\right)^{\circ\left(a_{1}-1\right)}, \xi_{1}, \ldots, \xi_{r}\right\rangle_{\pi^{\prime} \circ \pi} \\
= & \left\langle\left(\pi^{*} \ell_{1}\right)^{\circ a_{1}}, \xi_{1}, \ldots, \xi_{r}\right\rangle_{\pi^{\prime} \circ \pi} \\
& +\left\langle\mathbf{P}_{1},\left(\pi^{*} \ell_{1}\right)^{\circ\left(a_{1}-1\right)}, \xi_{1}, \ldots, \xi_{r}\right\rangle_{\pi^{\prime} \circ \pi}
\end{aligned}
$$

for any line bundles $\xi_{1}, \ldots, \xi_{r}$ in $\operatorname{Pic}\left(\mathcal{C}_{g, M}\right)$, where $a_{1}+r=m+2$. Now if there are $a_{2}$ indices $i$ with $\xi_{i}=L_{2}$, then we can do the same with $L_{2}$ as we did with $L_{1}$. This gives four terms a priori, but one of them is $\left\langle\mathbf{P}_{1},\left(\pi^{*} \ell_{1}\right)^{\circ\left(a_{1}-1\right)}, \mathbf{P}_{2},\left(\pi^{*} \ell_{2}\right)^{\circ\left(a_{2}-1\right)}, \ldots\right\rangle_{\pi^{\prime} \circ \pi}$ and this vanishes by Prop. 3(a). Continuing, we find

$$
\begin{aligned}
& \left\langle L_{1}^{\circ a_{1}}, \ldots, L_{M}^{\circ a_{M}}, L_{M+1}^{\circ a}\right\rangle_{\pi^{\prime} \circ \pi} \\
& =\left\langle\left(\pi^{*} \ell_{1}\right)^{\circ a_{1}}, \ldots,\left(\pi^{*} \ell_{M}\right)^{\circ a_{M}}, L_{M+1}^{\circ a}\right\rangle_{\pi^{\prime} \circ \pi} \\
& \quad+\sum_{i=1}^{M}\left\langle\mathbf{P}_{i},\left(\pi^{*} \ell_{1}\right)^{\circ a_{1}}, \ldots,\left(\pi^{*} \ell_{i}\right)^{\circ\left(a_{i}-1\right)}, \ldots,\left(\pi^{*} \ell_{M}\right)^{\circ a_{M}}, L_{M+1}^{\circ a}\right\rangle_{\pi^{\prime} \circ \pi}
\end{aligned}
$$

for any $a_{i}, a \geq 0$. We have to evaluate this in two cases, when $a=0$ and when $a=1$.

If $a=0$, then the first term in (8) vanishes, because each of the arguments of the Deligne product is a pull-back under $\pi$. For the second term, we note that

$$
\left\langle\mathbf{P}_{i}, \pi^{*} \xi_{1}, \ldots, \pi^{*} \xi_{m+1}\right\rangle_{\pi^{\prime} \circ \pi}=\left\langle\xi_{1}, \ldots, \xi_{m+1}\right\rangle_{\pi^{\prime}}
$$

for any $\xi_{1}, \ldots, \xi_{m+1} \in \operatorname{Pic}\left(\mathcal{M}_{g, M}\right)$. (This follows from (DP3), because $P_{i}$ is a section of $\pi$.) This gives Eq. (6) and hence Proposition 4 (string equation).

If $a=1$, then the second term of (8) vanishes, because

$$
\left\langle\mathbf{P}_{i}, \pi^{*} \xi_{1}, \ldots, \pi^{*} \xi_{m}, L_{M+1}\right\rangle_{\pi^{\prime} \circ \pi}=\left\langle\left\langle\mathbf{P}_{i}, L_{M+1}\right\rangle_{\pi}, \xi_{1}, \ldots, \xi_{m}\right\rangle_{\pi^{\prime}}=0
$$

for any $\xi_{1}, \ldots, \xi_{m} \in \operatorname{Pic}\left(\mathcal{M}_{g, M}\right)$, by Prop. 1 (a) and Prop. 3 (c). The first term in (8) is equal to the right-hand side of (7) by Eq. (5) and Prop. 3 (d). This proves Eq. (7) and hence Proposition 6 (dilaton equation). 


\section{Proof of the Main Theorem}

Since the recursion formula and initial values given in Propositions 4 and 5 determine the elements $T_{N}\left(a_{1}, \ldots, a_{N+m}\right) \in \operatorname{Pic}\left(\mathcal{M}_{g, N}\right)$ uniquely, we can prove Theorem 1 by showing that the elements $T_{N}\left(a_{1}, \ldots, a_{N+m}\right)$ defined by (2) and (3) satisfy these three equations. A direct proof of this is possible, but rather complicated, involving a series of lemmas about elementary symmetric polynomials and multinomial coefficients. This proof can be simplified considerably by a judicious use of generating functions, but remains quite complicated. A much simpler proof is obtained using the following trick. By the substitution $t=x /(1+x)$ and Euler's formula for the beta integral (or simply by integration by parts and induction on $\alpha$ and $\beta$ ) we see that

$$
\int_{0}^{\infty} \frac{x^{\alpha} d x}{(x+1)^{\alpha+\beta+2}}=\int_{0}^{1} t^{\alpha}(1-t)^{\beta} d t=\frac{\alpha ! \beta !}{(\alpha+\beta+1) !}
$$

for any integers $\alpha, \beta \geq 0$. Hence, if we define a polynomial $F(x)=F_{d_{1}, \ldots, d_{m}}(x)$ by

$$
F(x)=\prod_{j=1}^{m}\left(x+d_{j}\right)=\sum_{n=0}^{m} \sigma_{n} x^{m-n}, \quad \sigma_{n}=\sigma_{n}\left(d_{1}, \ldots, d_{m}\right),
$$

then we have

$$
\int_{0}^{\infty} \frac{F(x) d x}{(x+1)^{\widetilde{N}+m+1}}=\sum_{n=0}^{m} \sigma_{n} \frac{(m-n) !(\tilde{N}+n-1) !}{(\widetilde{N}+m) !}=\frac{(\widetilde{N}-1) !}{(\widetilde{N}+m) !} C_{1}(\mathbf{d})
$$

and

$$
\int_{0}^{\infty} \frac{x F(x) d x}{(x+1)^{\tilde{N}+m+1}}=\sum_{n=0}^{m} \sigma_{n} \frac{(m-n+1) !(\tilde{N}+n-2) !}{(\widetilde{N}+m) !}=\frac{\widetilde{N}(\tilde{N}-2) !}{(\widetilde{N}+m) !} C_{2}(\mathbf{d})
$$

with $C_{v}(\mathbf{d})=C_{v}\left(\widetilde{N} ; d_{1}, \ldots, d_{m}\right)$ as in Eq. (3) (or the first remark after Theorem 1 if $\widetilde{N}$ is 0 or 1$)$. In particular, we have

$$
\begin{gathered}
C_{1}(\tilde{N} ; \underbrace{1, \ldots, 1}_{m})=\frac{(\widetilde{N}+m) !}{(\widetilde{N}-1) !} \int_{0}^{\infty} \frac{d x}{(x+1)^{\tilde{N}+1}}=\frac{(\widetilde{N}+m) !}{\widetilde{N} !}, \\
C_{2}(\widetilde{N} ; \underbrace{1, \ldots, 1}_{m})=\frac{(\widetilde{N}+m) !}{\widetilde{N}(\widetilde{N}-2) !} \int_{0}^{\infty} \frac{x d x}{(x+1)^{\tilde{N}+1}}=\frac{(\widetilde{N}+m) !}{\widetilde{N} \cdot \widetilde{N} !}, \\
C_{2}(\widetilde{N} ; 2, \underbrace{1, \ldots, 1}_{m-1})=\frac{(\widetilde{N}+m) !}{\widetilde{N}(\widetilde{N}-2) !} \int_{0}^{\infty} \frac{x(x+2) d x}{(x+1)^{\widetilde{N}+2}}=\frac{2(\tilde{N}+m) !}{(\widetilde{N}+1) !},
\end{gathered}
$$

so Eq. (2) in the two cases when all $d_{i}$ are strictly positive reduces to

$$
\begin{aligned}
& T_{N, m}(1, \underbrace{0, \ldots, 0}_{N-1} ; \underbrace{1, \ldots, 1}_{m}) \\
& \quad=C_{1}(\widetilde{N} ; 1, \ldots, 1)\left(\ell_{1}-\frac{\Delta}{\widetilde{N}}\right)+C_{2}(\widetilde{N} ; 1, \ldots, 1) \Delta=\frac{(\widetilde{N}+m) !}{\widetilde{N} !} \ell_{1}
\end{aligned}
$$


and

$$
\begin{aligned}
& T_{N, m}(\underbrace{0, \ldots, 0}_{N} ; 2, \underbrace{1, \ldots, 1}_{m-1}) \\
& =\frac{1}{2 !} C_{2}(\tilde{N} ; 2,1, \ldots, 1) \Delta=\frac{(\widetilde{N}+m) !}{(\widetilde{N}+1) !} \Delta,
\end{aligned}
$$

in accordance with the initial values in Proposition 5.

To prove the recursion formula of Proposition 4 (string equation), we will show that it is equivalent to a pair of recurrences for the coefficients $C_{\nu}(\mathbf{d})$ (Eq. (11) below) and then prove these recurrences using the integral representation (9). Denote the right-hand side of (2) by $t_{N, m}\left(a_{1}, \ldots, a_{N+m}\right)$ or $t_{N, m}\left(a_{1}, \ldots, a_{N} ; d_{1}, \ldots, d_{m}\right)$, with $d_{j}=a_{N+j}$ for $1 \leq j \leq m$. Since we want $T_{N, m}\left(a_{1}, \ldots, a_{N+m}\right)=t_{N, m}\left(a_{1}, \ldots, a_{N+m}\right) / \prod_{i=1}^{N+m} a_{i}$ !, we have to prove the recursion

$$
t_{N, m+1}\left(a_{1}, \ldots, a_{N+m}, 0\right)=\sum_{i=1}^{N+m} a_{i} t_{N, m}\left(a_{1}, \ldots, a_{i}-1, \ldots, a_{N+m}\right) .
$$

(The extra factor $a_{i}$ in front of $t_{N, m}\left(a_{1}, \ldots, a_{i}-1, \ldots, a_{N+m}\right)$ comes from the change in $\prod_{i=1}^{N+m} a_{i}$ ! when $a_{i}$ is decreased by 1 .) Now again separating the $a_{i}(1 \leq i \leq N)$ and the $d_{j}=a_{N+j}(1 \leq j \leq m)$, we can write this out more explicitly as

$$
\begin{aligned}
& t_{N, m+1}\left(a_{1}, \ldots, a_{N} ; d_{1}, \ldots, d_{m}, 0\right) \\
& =\sum_{i=1}^{N} a_{i} t_{N, m}\left(a_{1}, \ldots, a_{i}-1, \ldots, a_{N} ; d_{1}, \ldots, d_{m}\right) \\
& \quad+\sum_{j=1}^{m} d_{j} t_{N, m}\left(a_{1}, \ldots, a_{N} ; d_{1}, \ldots, d_{j}-1, \ldots, d_{m}\right) .
\end{aligned}
$$

We can write the definition of $t_{N, m}\left(a_{1}, \ldots, a_{N} ; d_{1}, \ldots, d_{m}\right)$ in an abbreviated notation as

$$
t_{N, m}\left(a_{1}, \ldots, a_{N} ; d_{1}, \ldots, d_{m}\right)=C_{1}(\mathbf{d}) \sum_{k=1}^{N} a_{k} \widehat{\ell}_{k}+C_{2}(\mathbf{d}) \Delta,
$$

where $C_{v}(\mathbf{d})=C_{v}\left(\tilde{N} ; d_{1}, \ldots, d_{m}\right)$ as before $(\widetilde{N}$ does not change when we change $m$ by 1 , so can be omitted from the notation) and where $\widehat{\ell}_{k}$ is the element $\ell_{k}-\Delta / N$ of $\operatorname{Pic}\left(\mathcal{M}_{g, N}\right) \otimes \mathbb{Q}$. Then the left-hand side of (10) equals

$$
C_{1}(\mathbf{d}, 0) \sum_{i=1}^{N} a_{k} \widehat{\ell}_{k}+C_{2}(\mathbf{d}, 0) \Delta,
$$

while the right-hand side equals

$$
\begin{aligned}
\sum_{i=1}^{N} a_{i} & {\left[C_{1}(\mathbf{d}) \sum_{k=1}^{N}\left(a_{k}-\delta_{k i}\right) \widehat{\ell}_{k}+C_{2}(\mathbf{d}) \Delta\right] } \\
& +\sum_{j=1}^{m} d_{j}\left[C_{1}\left(d_{1}, \ldots, d_{j}-1, \ldots, d_{m}\right) \sum_{k=1}^{N} a_{k} \widehat{\ell}_{k}+C_{2}\left(d_{1}, \ldots, d_{j}-1, \ldots, d_{m}\right) \Delta\right]
\end{aligned}
$$




$$
\begin{gathered}
=\sum_{k=1}^{N}\left[C_{1}(\mathbf{d})\left(\sum_{i=1}^{N} a_{i}-1\right)+\sum_{j=1}^{m} d_{j} C_{1}\left(d_{1}, \ldots, d_{j}-1, \ldots, d_{m}\right)\right] a_{k} \widehat{\ell}_{k} \\
+\left[C_{2}(\mathbf{d})\left(\sum_{i=1}^{N} a_{i}\right)+\sum_{j=1}^{m} d_{j} C_{2}\left(d_{1}, \ldots, d_{j}-1, \ldots, d_{m}\right)\right] \Delta .
\end{gathered}
$$

Comparing these two expressions, and recalling that $\sum_{i=1}^{N} a_{i}=m+2-\sigma_{1}(\mathbf{d})$ (because the sum of all the indices $a_{1}, \ldots, a_{N}, d_{1}, \ldots, d_{m}, 0$ in Eq. (10) must equal $m+2$ ), we find that the theorem will follow from the two identities:

$$
\begin{aligned}
& C_{1}(\mathbf{d}, 0)=\left(m+1-\sigma_{1}\right) C_{1}(\mathbf{d})+\sum_{j=1}^{m} d_{j} C_{1}\left(d_{1}, \ldots, d_{j}-1, \ldots, d_{m}\right), \\
& C_{2}(\mathbf{d}, 0)=\left(m+2-\sigma_{1}\right) C_{2}(\mathbf{d})+\sum_{j=1}^{m} d_{j} C_{2}\left(d_{1}, \ldots, d_{j}-1, \ldots, d_{m}\right) .
\end{aligned}
$$

To prove the first of these, we use Eq. (9a). Replacing $\mathbf{d}=\left(d_{1}, \ldots, d_{m}\right)$ by $(\mathbf{d}, 0)=$ $\left(d_{1}, \ldots, d_{m}, 0\right)$ increases $m$ by 1 and replaces the polynomial $F(x)$ by $x F(x)$, whereas replacing $\mathbf{d}$ by $\left(d_{1}, \ldots, d_{j}-1, \ldots, d_{m}\right)$ leaves $m$ unchanged and replaces $F(x)$ by $F(x)\left(x+d_{j}-1\right) /\left(x+d_{j}\right)$. Therefore substituting (9a) into (11a) and dividing both sides by $(\widetilde{N}+m) ! /(\widetilde{N}-1)$ ! gives

$$
\begin{aligned}
& (\tilde{N}+m+1) \int_{0}^{\infty} \frac{x F(x) d x}{(x+1)^{\widetilde{N}+m+2}} \\
& =\int_{0}^{\infty}\left[m+1+\sum_{j=1}^{m} d_{j}\left(-1+\frac{x+d_{j}-1}{x+d_{j}}\right)\right] \frac{F(x) d x}{(x+1)^{\widetilde{N}+m+1}}
\end{aligned}
$$

as the identity to be proved. But this is immediate by integration by parts, since the expression in square brackets equals $1+\sum_{j=1}^{m} \frac{x}{x+d_{j}}=1+x \frac{F^{\prime}(x)}{F(x)}$. The proof of Eq. (11b) is exactly the same, using (9b) instead of (9a), with $F(x)$ replaced by $x F(x)$. This completes the proof of the theorem.

\section{Final Remark}

A formula very similar to Eq. (2) (in the case when all $a_{i}=0$ ) appears in $\$ 4.6$ of [8], but in a somewhat different situation: the formula there is for the moduli space of curves of genus 1 with $N$ marked points and deals with the Gromov-Witten invariants, which are integers, whereas our formula is for arbitary genus (although in the final result the genus does not appear except in the shift from $N$ to $\widetilde{N}$ ) and gives the Deligne products, which take values in $\operatorname{Pic}\left(\mathcal{M}_{g, N}\right)$. Both proofs are based on the string and dilaton equations, which are valid in both contexts. This suggests a possible common generalization. Our situation concerns codimension one cycles, while Gromov-Witten invariants have to do with zero-dimensional cycles. It therefore seems reasonable to ask whether (2) and the equation in [8] are special cases of a more general result valid for intermediate dimensions, for which the string and dilaton equations still hold. 
Open Access This article is distributed under the terms of the Creative Commons Attribution Noncommercial License which permits any noncommercial use, distribution, and reproduction in any medium, provided the original author(s) and source are credited.

\section{References}

1. Arbarello, E., Cornalba, M.: The Picard groups of the moduli spaces of curves. Topology 26(2), 153-171 (1987)

2. Deligne, P.: Le déterminant de la cohomologie. In: Current trends in arithmetic algebraic geometry, Contemporary Math. Vol. 67, Providence, RI: Amer. Math. Soc., pp. 93-178, 1987

3. Deligne, P., Mumford, D.: The irreducibility of the space of curves of given genus. IHES Publ. Math. 36, 75-109 (1969)

4. Harer, J.: The second homology group of the mapping class group of an orientable surface. Invent. Math. 72(2), 221-239 (1983)

5. Kaufmann, R., Manin, Yu., Zagier, D.: Higher Weil-Petersson volumes of moduli spaces of stable n-pointed curves. Commun. Math. Phys. 181(3), 763-787 (1996)

6. Knudsen, F.: The projectivity of the moduli space of stable curves, II. Math. Scand. 52, 161-199 (1983)

7. Knudsen, F., Mumford, D.: The projectivity of the moduli space of stable curves. I. Math. Scand. 39, 19-55 (1976)

8. Lando, S.K., Zvonkin, A.K.: Graphs on Surfaces and Their Applications, In: Encyclopedia of Mathematical Sciences, Berlin-Heidelberg: Springer-Verlag, 2004

9. Takhtajan, L., Zograf, P.: The Selberg zeta function and a new Kähler metric on the moduli space of punctured Riemann surfaces. J. Geo. Phys. 5, 551-570 (1988)

10. Takhtajan, L., Zograf, P.: A local index theorem for families of $\bar{\partial}$-operators on punctured Riemann surfaces and a new Kähler metric on their moduli spaces. Commun. Math. Phys. 137, 399-426 (1991)

11. Weng, L.: Hyperbolic Metrics, Selberg Zeta Functions and Arakelov Theory for Punctured Riemann Surfaces. Lecture Note Series in Mathematics 6, Osaka: Osaka University, 1998

12. Weng, L.: $\Omega$-admissible theory, II.. Math. Ann. 320, 239-283 (2001)

Communicated by L. Takhtajan 